\title{
Degenerates with Dusty Disks: White Dwarfs and Cataclysmic Variables in the Infrared
}

\author{
D. W. Hoard $\mathbf{H}^{1,2}$ \\ ${ }^{1}$ Spitzer Science Center, California Institute of Technology \\ Pasadena, CA, USA \\ ${ }^{2}$ Eureka Scientific, Inc. \\ Oakland, CA, USA \\ email: donald.hoard@gmail.com
}

\begin{abstract}
Recent infrared observations, particularly from the Spitzer Space Telescope and Widefield Infrared Survey Explorer, of white dwarfs, cataclysmic variables and other interacting compact binaries, have revealed the presence of dust in many systems. I briefly review the discovery and observational properties of dust around white dwarfs and cataclysmic variables.
\end{abstract}

Keywords. Stars: dwarf novae; novae, cataclysmic variables; dust, extinction; Infrared: stars

\section{Introduction}

Dust disks are common in a wide variety of astrophysical situations, ranging from young stars to central engines of quasars. The first white dwarf (WD) with a dust disk, G29-38, was discovered by Zuckerman \& Becklin (1987) via its infrared (IR) excess. Jura (2003) developed a model for the origin of WD dust disks involving the tidal disruption of a comet or asteroid that was perturbed into the WD Roche lobe, likely due to the gravitational influence of a remnant planetary system. By the end of 2010, only 20 dusty WDs were known (see Table 5.1 in Farihi 2011), largely owing to sensitive IR observations obtained with the Spitzer Space Telescope. Recently, the WISE IR Excesses around Degenerates (WIRED) Survey (Debes et al. 2011b), which cross-correlated the Sloan Digital Sky Survey Data Release 7 preliminary WD Catalog (Kleinman 2010) with the Wide-Field Infrared Survey Explorer all-sky photometry at 3.4, 4.6, 12, and $22 \mu \mathrm{m}$, has more than tripled the number of known dusty WDs (see Figure 1). The WIRED Survey is sensitive to dusty debris around WDs out to distances of $\sim 100 \mathrm{pc}$, well beyond the completeness level of local WDs.

One of the most exciting topics in astronomy today is the study of planets around other stars. The more than 800 exoplanets discovered to date have shown that planetary systems exist around a range of host stars and in a variety of architectures that have radically affected our understanding of how planets form. The potential for discovery of planets in orbit around giant stars, subgiants, WDs, and neutron stars motivates the study of how planetary systems change during post-main sequence evolution. Dusty debris disks around WDs are unique laboratories for studying the late evolutionary stages of planetary systems, and provide insight into the future of the Solar System. As disk material is accreted onto the WD, it can be used to study the chemical composition of the extrasolar equivalent of Solar System bodies from which it originated (i.e., asteroids, comets, planetesimals). Figure 1 shows the spectral energy distribution (SED) of the WD GALEX 1931+0117, demonstrating the characteristic profile of a WD dust disk. 


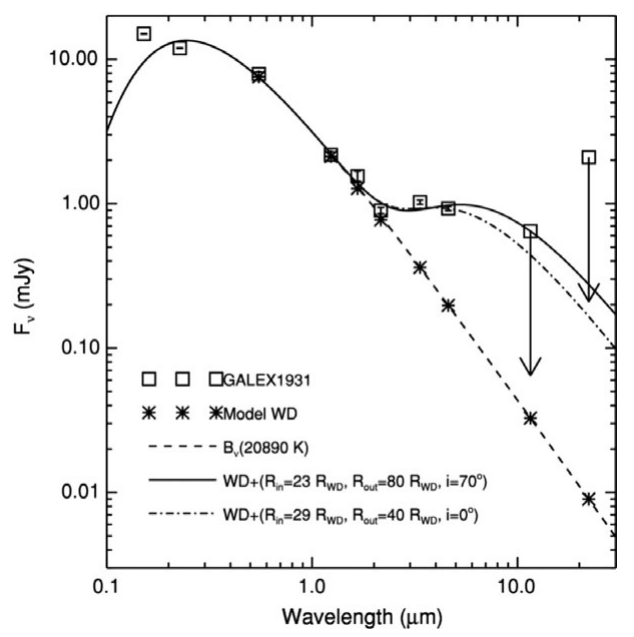

Figure 1. UV, V-band, near-IR (2MASS JHK) and WISE photometry (squares) of the dusty WD GALEX1931+0117, compared to synthetic photometry of a similar model WD (asterisks), a black body with $T_{\text {eff }}=20,890 \mathrm{~K}$ (dashed line), and two different dust disk models assuming a flat optically thick disk (see Debes et al. 2011a).

\section{The White Dwarf - Dust - Planet Connection}

Predating the discovery of dust around WDs, it was known that a small fraction of WDs show absorption lines of metals in their optical and UV photospheric spectra (e.g., Lacombe et al. 1983, Shipman \& Greenstein 1983). Gravitational settling times in hydrogen-rich (DA) WD atmospheres are very short (a few days to $\lesssim 1000$ yr), so metals quickly diffuse out of the photosphere. The observed metals were thought to be supplied by ongoing accretion from the ISM (Sion et al. 1990). This explanation was problematic for several reasons; notably, explaining the relative elemental abundances of the accreted material, which do not match equilibrium ISM values (see Section 5.6.6 in Farihi 2011).

There is an intimate link between humans and WDs, for the future fate of our Sun is to evolve into a WD. The effects of this process on the Solar System at large are issues of intrinsic interest to the inhabitants of the Solar System, since they will determine its future contents and suitability for habitation. Planets in initially wide orbits (radii > a few AU) can survive as a central star becomes a WD (Duncan \& Lissauer 1998), although many objects in the inner system are destroyed through engulfment or evaporation (Villaver \& Livio 2007, 2009; Nordhaus et al. 2010). To form dust disks, surviving rocky planetesimals must be perturbed (presumably by a planet) on timescales of Myr-Gyr, and be tidally disrupted by the WD (Jura 2003, 2008).

Evidence for a link between dusty WDs and remnant planetary systems is mounting. Order of magnitude estimates for the expected number of metal-polluted WDs (Debes \& Sigurdsson 2002) are consistent with observations, and similar to the frequency of giant planets around early type stars (Johnson et al. 2010) and estimated frequency of closein Earth mass planets (Howard et al. 2010). Also, the relative abundances of accreted metals in several WDs are similar to that of the Moon and terrestrial planets (Zuckerman et al. 2007, Gänsicke et al. 2012). Detailed inspection of abundances in metal-rich WDs provides a unique insight into the composition of extrasolar planetesimals. Analyses of two metal-polluted WDs (GD61, NLTT 43806) suggest that the accreted dust came from an asteroid whose origin was in the Ca-rich and possibly water-rich lithosphere of a differentiated planet (Farihi et al. 2011, Zuckerman et al. 2011). 


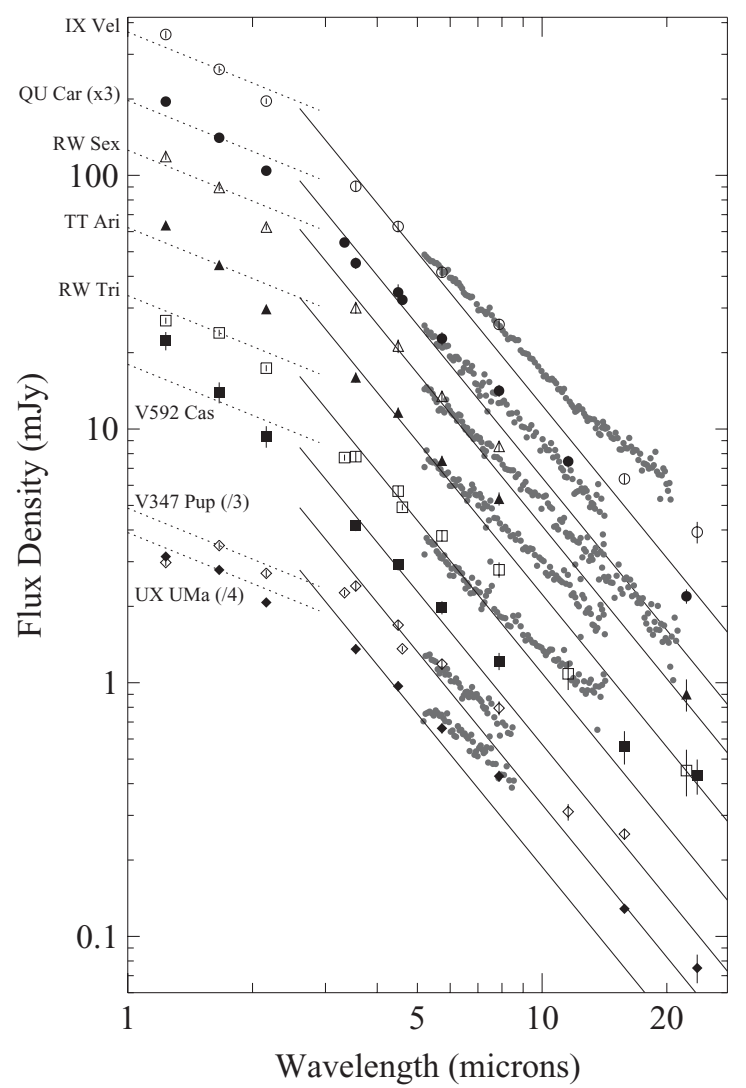

Figure 2. Infrared SEDs of 8 novalike CVs. Large points are 2MASS, WISE, and Spitzer-IRAC, -IRS-PUI, and -MIPS photometry; small points are Spitzer-IRS spectra. Dotted lines show the "disk spectrum" portion of a standard steady-state accretion disk SED (Frank, King, \& Raine 1992), scaled to the H-band point, while the solid lines show the Rayleigh-Jeans tail portion of the accretion disk spectrum, scaled to the mean of the IRAC channel 1 and 2 points. The excess above the latter disk component indicates the presence of dust.

\section{Dust in Cataclysmic Variables}

Cataclysmic variables (CVs) have been observed in every Spitzer Guest Observer cycle, probing longer wavelengths, at higher sensitivity, than in any previous IR observations of CVs. A remarkable early discovery was the nearly ubiquitous presence of an IR excess signature attributable to thermal emission from dust (e.g., Howell et al. 2006, Brinkworth et al. 2007; see Figure 2). In some cases (e.g., WZ Sge, Z Cha), the dust in CVs is in circumstellar orbit around the WD. It survives the harsh environment near the hot WD by lurking in the shadow of the accretion disk, near the periphery of the Roche lobe (Howell et al. 2008). The observational signatures of the presence and distribution of this dust are an IR excess accompanied by a mid-IR light curve displaying an eclipse that is significantly longer in duration than the optical eclipse (Howell et al. 2008). In other cases (e.g., V592 Cas), the dust is in a circumbinary disk around the entire CV (Hoard et al. 2009). Circumbinary disks were suggested as possible sources of additional angular momentum loss contributing to the secular evolution of CVs (Spruit \& Taam 2001; Taam et al. 2003; Willems et al. 2005, 2007); however, the Spitzer results imply total dust masses many orders of magnitude too small to be effective in that respect 
(Hoard et al. 2009). Lingering questions about the dust remain: what is its composition, size, shape, etc.? What is its origin? How rapidly is it replenished and/or destroyed?

An important, and discriminating, distinction between the two cases for the distribution of dust in CVs (circumstellar vs. circumbinary) emerges from the dust disk models used to fit the IR excess. For example, in WZ Sge (Howell et al. 2008), the dust extends from $\mathrm{R}_{\text {in }} \approx 11 \mathrm{R}_{\mathrm{wd}}$, corresponding to the dust sublimation temperature $(\approx 1500 \mathrm{~K})$, to $\mathrm{R}_{\text {out }} \approx 30 \mathrm{R}_{\mathrm{wd}}$, which is constrained by the lowest temperature consistent with the IR excess $(\approx 700 \mathrm{~K})$. This sharp cutoff in temperature corresponds to a radius close to the WD Roche lobe, consistent with the dust extending outward from the edge of the accretion disk. In contrast, although the dust disk model in V592 Cas (Hoard et al. 2009) is constrained to also start at a specific radius (corresponding to the tidal truncation radius outside the $\mathrm{CV}$ ), the IR excess out to $24 \mu \mathrm{m}$ does not constrain the outer (i.e., low) dust temperature. This situation is consistent with a dust distribution with no sharp outer boundary that, instead, merges into the ambient ISM surrounding the CV.

\section{Acknowledgements}

I want to acknowledge and thank my many collaborators on the various projects summarized here, especially J. Debes, S. Howell, K. Long, P. Szkody, and S. Wachter. This work was supported by NASA through an award issued by JPL/Caltech.

\section{References}

Brinkworth, C. S., Hoard, D. W., Wachter, S., et al. 2007, ApJ, 659, 1541

Debes, J. H., Hoard, D. W., Kilic, M., et al. 2011a, ApJ, 729, 4

Debes, J. H., Hoard, D. W., Wachter, S., et al. 2011b, ApJS, 197, 38

Debes, J. H. \& Sigurdsson, S. 2002, ApJ, 572, 556

Duncan, M. J. \& Lissauer, J. J. 1998, Icarus, 134, 303

Farihi, J. 2011, in White Dwarf Atmospheres and Circumstellar Environments, ed. D. W. Hoard (Wiley: Berlin), 117-171

Farihi, J., Brinkworth, C. S., Gänsicke, B. T., et al. 2011, ApJL, 728, L8

Frank, J. R., King, A. R., \& Raine, D. J. 1992, Accretion Power in Astrophysics (2nd Edition)

(Cambridge: Cambridge University Press), p. 79

Gänsicke, B. T., Koester, D., Farihi, J., et al. 2012, MNRAS, 424, 333

Hoard, D. W., Kafka, S., Wachter, S., et al. 2009, ApJ, 693, 236

Howard, A. W., Marcy, G. W., Johnson, J. A., et al. 2010, Science, 330, 653

Howell, S. B., Brinkworth, C., Hoard, D. W., et al. 2006, ApJL, 646, L65

Howell, S. B., Hoard, D. W., Brinkworth, C. S., et al. 2008, ApJ, 685, 418

Johnson, J. A., Aller, K. M., Howard, A. W., \& Crepp, J. R. 2010, PASP, 122, 905

Jura, M. 2003, ApJL, 584, L91

Jura, M. 2008, $A J, 135,1785$

Kleinman, S. J. 2010, American Institute of Physics Conference Series, 1273, 156

Lacombe, P., Wesemael, F., Fontaine, G., et al. 1983, ApJ, 272, 660

Nordhaus, J., Spiegel, D. S., Ibgui, L., et al. 2010, MNRAS, 408, 631

Shipman, H. L. \& Greenstein, J. L. 1983, ApJ, 266, 761

Sion, E. M., Kenyon, S. J., \& Aannestad, P. A. 1990, ApJS, 72, 707

Spruit, H. C. \& Taam, R. E. 2001, ApJ, 548, 900

Taam, R. E., Sandquist, E. L., \& Dubus, G. 2003, ApJ, 592, 1124

Villaver, E. \& Livio, M. 2007, ApJ, 661, 1192

Villaver, E. \& Livio, M. 2009, ApJ, 705, L81

Willems, B., Kolb, U., Sandquist, E. L., et al. 2005, ApJ, 635, 1263

Willems, B., Taam, R. E., Kolb, U., et al. 2007, ApJ, 657, 465

Zuckerman, B. \& Becklin, E. E. 1987, Nature, 330, 138

Zuckerman, B., Koester, D., Dufour, P., et al. 2011, ApJ, 739, 101

Zuckerman, B., Koester, D., Melis, C., et al. 2007, ApJ, 671, 872 